\title{
Biological activity detection method using MIMO system
}

\author{
Masaki Nango $^{1 \mathrm{a})}$, Naoki Honma ${ }^{1}$, Kentaro Nishimori ${ }^{2}$, \\ and Hiroaki Sato ${ }^{1}$ \\ ${ }^{1}$ Graduate School of Engineering, Iwate University 4-3-5 Ueda, Morioka, 020- \\ 8551, Japan \\ ${ }^{2}$ Graduate School of Science \& Technology, Niigata University 2-8050, Ikarashi, \\ Niigata, 950-2181, Japan \\ a)t2311045@iwate-u.ac.jp
}

\begin{abstract}
This letter proposes two biological activity detection methods using MIMO system and compares them. These methods detect the biological activity of human by using the time-variant MIMO channel and improve the detection sensitivity by using the multiantennas. Experiment and simulation results demonstrate the proposed method can improve the detection sensitivity than the detection method using SISO system.
\end{abstract}

Keywords: MIMO, biological activity, microwave, welfare, sensor

Classification: Antennas and Propagation

\section{References}

[1] Y. J. An, B. J. Jang, and J. G. Yook, "Detection of human vital signs and estimation of direction of arrival using multiple doppler radars," Journal of The Korean Institute of Electromagnetic Engineering and Science, vol. 10, no. 4, pp. 250-255, Dec. 2010.

[2] R. R. Fletcher and S. Kulkarni, "Clip-on wireless wearable microwave sensor for ambulatory cardiac monitoring," $E M B C$ 2010, vol. 1, pp. 365369, Aug. 2010.

[3] N. Petrochilos, M. Rezk, A. Host-Madsen, V. Lubecke, and O. BoricLubecke, "Blind separation of human heartbeats and respiration by the use of a doppler radar remote sensing," ICASSP 200\%, vol. 1, pp. I-333I-336, April 2007.

[4] D. Samardzija, B. K. Park, O. Boric-Lubecke, V. Lubecke, A. HostMadsen, and T. Sizer, "Experimental evaluation of multiple antenna techniques for remote sensing of physiological motion," 2007 IEEE/ MTT-S International Microwave Symposium, vol. 1, pp. 1735-1738, June 2007.

[5] O. Boric-Lubecke, V. M. Lubecke, A. Host-Madsen, D. Samardzija, and K. Cheung, "Doppler radar sensing of multiple subjects in single and multiple antenna systems," Telecommunications in Modern Satellite, Cable and Broadcasting Services, 2005, vol. 1, pp. 7-11, Sept. 2005.

[6] J. G. Kim, S. H. Sim, S. Cheon, and S. Hong, "24 GHz circularly polarized doppler radar with a single antenna," Microwave Conference, 2005 European, vol. 2, pp. 1383-1386, Oct. 2005.

[7] D. Nagae and A. Mase, "Measurement of heart rate variability and stress evaluation by using microwave reflectometric vital signal sensing," Re- 
view of Scientific Instruments, vol. 81, pp. 094301-1-094301-10, Sept. 2010.

[8] H. Avagyan, A. Hakhoumian, H. Hayrapetyan, N. Pogosyan, and T. Zakaryan, "Portable non-contact microwave doppler radar for respiration and heartbeat sensing," Armenian Journal of Physics, vol. 5, no. 1, pp. 814, April 2012.

[9] K. Higashikatsuragi, Y. Nakahata, I. Matsunami, and A. Kajiwara, "Remote respiration monitoring using ultra-wideband microwave sensor," IEEJ Transactions on Electronics, Information and Systems (in Japanese), vol. 129, no. 6, pp. 1056-1061, 2009.

[10] K. Ota, M. Otsu, Y. Ota, and A. Kajiwara, "Elderly-care motion monitoring mensor using ultra-wideband radio," IEEJ Transactions on Electronics, Information and Systems (in Japanese), vol. 131, no. 9, pp. 15471552, 2011.

\section{Introduction}

A population aging and the increase of the elderly people living alone are ongoing issues in Japan. As a result, fall, tumble, and lonely death have become the social problems, and the need of safety confirmation system for elderly is growing. The use of video camera and the ECG (Electrocardiogram) can be considered for the safety confirmation. However, the former has a privacy problem and the latter has the problem that has physical and mental burden due to direct contact of the probe to the human body.

In order to solve these problems, the technologies using microwave have been studied $[1,2,3,4,5,6,7,8,9,10]$. The technologies do not violate privacy and can detect the vital sign without attaching probes. But there is a problem that the detection accuracy is degraded if the distance between a human body and the detector becomes greater [8].

In this letter, two biological activity detection methods, which can achieve high sensitivity by using MIMO (Multiple-Input Multiple-Output) system, are proposed. These methods detect the biological activity of human by exploiting the time-variant MIMO channel and improve the detection sensitivity by using the multi-antennas. Experiment and simulation results demonstrate the proposed method can improve the detection sensitivity more than the detection method using SISO (Single-Input Single-Output) system.

\section{Channel measurement conditions}

Fig. 1 shows the overview of the measurement system. In this study, the MIMO channels are measured by switching multiple transmitting and receiving antennas by using SPDT (Single-Pole Double-Throw) switches. $2 \times 2$ MIMO configuration where both transmitter and receiver have two horizontally arranged patch antennas are used. 6 patterns of the distance, $d$, between the antenna and the human body are tested, i.e., $d$ is $1.0 \mathrm{~m}, 2.0 \mathrm{~m}$, $3.0 \mathrm{~m}, 4.0 \mathrm{~m}, 5.0 \mathrm{~m}$, and $6.0 \mathrm{~m}$. The antenna height is $1.17 \mathrm{~m}$ which is as tall as the chest of subject. The spacing between transmitting antennas is 
0.5 wavelengths, and the spacing between transmitting and receiving arrays are 1.5 wavelength. Transmitted power is set to $0 \mathrm{dBm}$. $2.4 \mathrm{GHz}$ band CW signal is used; therefore, this measurement system can work with narrower bandwidth compared to the conventional studies [9, 10]. The time-variant channels are measured for 50 seconds when the subject is standing alone and facing to the antennas.

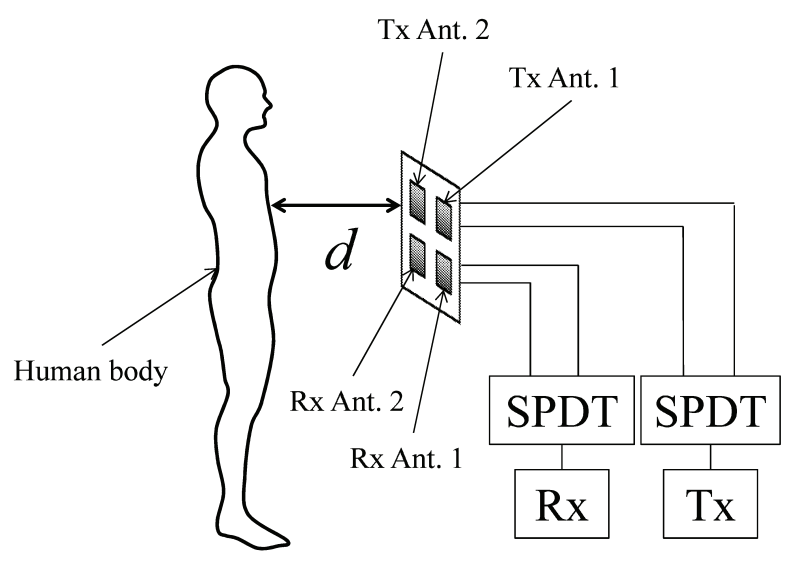

Fig. 1. Experimental configuration

\section{Proposed method}

The measured $m \times n$ time-variant MIMO channel is expressed as,

$$
\boldsymbol{H}(t)=\left(\begin{array}{ccc}
h_{11}(t) & \cdots & h_{1 n}(t) \\
\vdots & \ddots & \vdots \\
h_{m 1}(t) & \cdots & h_{m n}(t)
\end{array}\right),
$$

where, $h_{i j}$ is the complex channel response, and $t$ represents the time when the channel is observed. By Fourier transformation, the frequency response of this time-variant MIMO channel is expressed as,

$$
\boldsymbol{F}(f)=\left(\begin{array}{ccc}
F_{11}(f) & \cdots & F_{1 n}(f) \\
\vdots & \ddots & \vdots \\
F_{m 1}(f) & \cdots & F_{m n}(f)
\end{array}\right) .
$$

By eigenvalue decomposition, $\boldsymbol{F}(f)$ is expressed as,

$$
\boldsymbol{F}(f) \boldsymbol{F}(f)^{H}=\boldsymbol{U}(f) \boldsymbol{\lambda}(f) \boldsymbol{U}^{H}(f),
$$

where, $\{\cdot\}^{H}$ means the hermitian transpose. Also, $\boldsymbol{U}(f)$ represents the eigenvalue vectors and $\boldsymbol{\lambda}(f)$ is diagonal matrix representing eigenvalues. They are expressed as,

$$
\begin{gathered}
\boldsymbol{U}(f)=\left(\begin{array}{ccc}
\boldsymbol{u}_{11}(f) & \cdots & \boldsymbol{u}_{1 m}(f) \\
\vdots & \ddots & \vdots \\
\boldsymbol{u}_{m 1}(f) & \cdots & \boldsymbol{u}_{m m}(f)
\end{array}\right), \\
\boldsymbol{\lambda}(f)=\left(\begin{array}{ccc}
\lambda_{1}(f) & & 0 \\
& \ddots & \\
0 & & \lambda_{m}(f)
\end{array}\right) .
\end{gathered}
$$


Fig. 2 shows the frequency responses of time-variant SISO channels measured without the subject and with the subject when the distance, $d$, is $3.0 \mathrm{~m}$. This SISO channels are the components of the first row and first column of the MIMO channel matrices. This figure indicates that the influence of biological activity of human appears at low-frequency part.

In this letter, the following two methods are proposed and compared.

\section{(1)Method 1 :}

This is a method using sum of the energy spectral density of the timevariant channel as the evaluation function, which is expressed as,

$$
\rho_{1}=\max _{\substack{1 \leq i \leq m \\ 1 \leq j \leq n}} \int_{f_{1}}^{f_{2}}\left|F_{i j}(f)\right|^{2} d f .
$$

The channel with maximum energy is chosen, that is, this method will yield diversity effect.

\section{(2)Method 2 :}

This is a method using sum of the maximum eigenvalue at each frequency, and the evaluation function is expressed as,

$$
\rho_{2}=\int_{f_{1}}^{f_{2}} \lambda_{\max }(f) d f
$$

where, $f_{1}$ and $f_{2}$ are the frequency range containing the influence of human activities. In this letter, $f_{1}$ and $f_{2}$ are set to $0.02 \mathrm{~Hz}$ and $1.6 \mathrm{~Hz}$ respectively to observe the variation component influenced of biological activity except the DC component. $0.02 \mathrm{~Hz}$ is the lowest frequency which is determined by frequency resolution, and $1.6 \mathrm{~Hz}$ is the analyzable maximum frequency in this measurement since the acquisition frequency of the channel is $3.2 \mathrm{~Hz}$. If the evaluation function exceeds the threshold, the biological activity can be detected. The threshold value is set so that the false detection rate becomes $1 \%$ for both proposed methods. Where, the false detection is defined as the probability of evaluation function exceeding the threshold when there is no person.

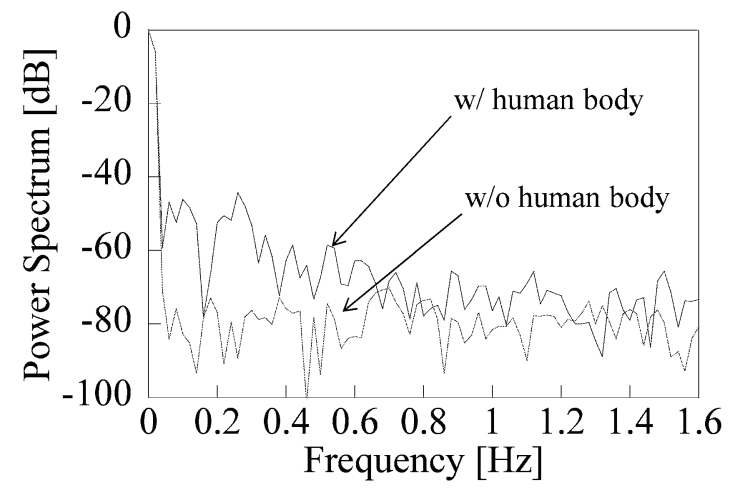

Fig. 2. Frequency response 


\section{Experimental result}

The detection rate is evaluated in the Monte Carlo simulation by adding the Gaussian noise to the measured channel. Detection rate is defined as (detection times) / (number of simulations). SNR (Signal to Noise Ratio) is defined as the ratio of the averaged signal power in static environment without the human to the noise power per antenna. Number of the simulations is set to 2000 .

Fig. 3(a) indicates the detection rate versus SNR when antenna distance, $d$, is $6.0 \mathrm{~m}$. In this result, the method 1 , which uses sum of the energy spectral density of the time-variant MIMO channel, improves the required SNR by about $3 \mathrm{~dB}$ for $90 \%$ detection rate compared with the method 1 using SISO channel. It is considered that this improvement is obtained by the diversity effect. And the method 2, which uses the maximum eigenvalue of frequency response matrix, shows the highest detection rate for any SNR, and improves the required SNR by about $5 \mathrm{~dB}$ for $90 \%$ detection rate compared with the method 1 using SISO channel. It is considered that this improvement is obtained by the effect of MRC (Maximum Ratio Combining).

Fig. 3(b) indicates the detection rate versus antenna distance when SNR is $10 \mathrm{~dB}$. In this result, the method 1 using MIMO channel improves the detec-

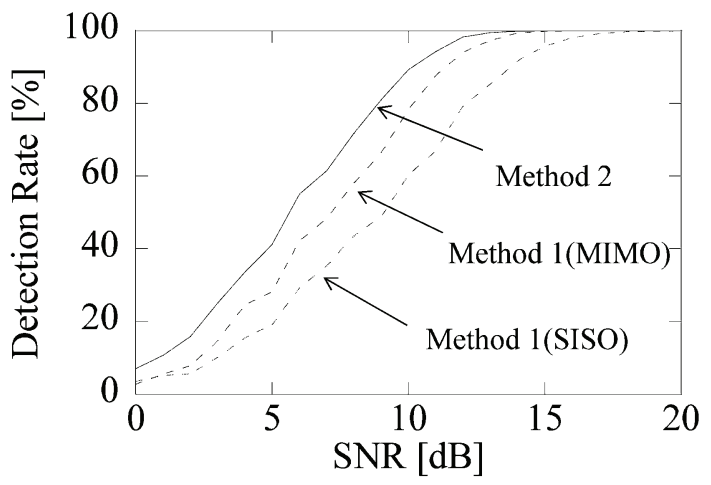

(a) Detection rate versus SNR when antenna distance, $d$, is $6.0 \mathrm{~m}$

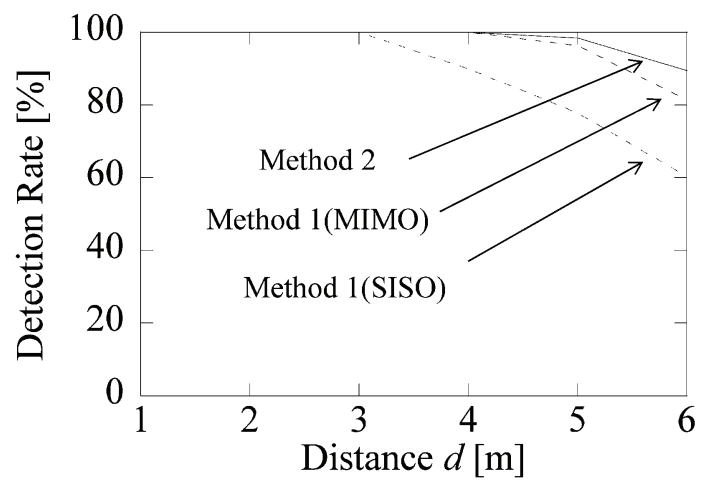

(b) Detection rate for distance when SNR is $10 \mathrm{~dB}$

Fig. 3. Experimental results 
tion rate by about $20 \%$ compared with method 1 using SISO channel when antenna distance is $6.0 \mathrm{~m}$. And the method 2 shows the highest detection rate for any antenna distances and improves the detection rate by about $30 \%$ compared with method 1 using SISO channel. Based on the results shown here, it is found the MIMO configuration yields higher sensitivity than SISO configuration, and the MRC scheme outperforms the diversity scheme.

\section{Conclusion}

This letter has proposed two biological activity detection methods with high sensitivity using MIMO system and compares their performances. The dependence of the detection rate on SNR was evaluated, and it is found that the proposed method 2, which uses the maximum eigenvalue of frequency response matrix, shows the highest detection rate among the methods discussed in this study. It is also found that the method 2 improves the required SNR by about $5 \mathrm{~dB}$ for $90 \%$ detection rate compared with method 1 using the SISO channel. The effect of the antenna distance was evaluated when SNR is $10 \mathrm{~dB}$, and it is found that the proposed method 2 improves the detection rate by about $30 \%$ compared with method 1 using SISO channel when antenna distance is $6.0 \mathrm{~m}$. From these results, it is found that the proposed method using the eigenvalue in MIMO channel is effective in detecting the biological activity. In this letter, the measurement was performed in a state in which the subject is standing. In the future, the evaluation in the different posture such as the lying subject will be investigated.

\section{Acknowledgements}

This research was partially supported by the Center for Revitalization Promotion of the Japan Science and Technology Agency (JST), and MEXT KAKENHI (23500549). 